\title{
2021 ACM PODS Alberto O. Mendelzon Test-of-Time Award
}

The ACM PODS Alberto O. Mendelzon Test-of-Time Award is awarded every year to a paper or a small number of papers published in the PODS proceedings ten years prior that had the most impact in terms of research, methodology, or transfer to practice over the intervening decade.

The PODS Executive Committee has appointed us to serve as the Award Committee for 2021. After careful consideration and having solicited external nominations and advice, we have selected the following paper as the award winner for 2021:

Tight bounds for $L_{p}$ samplers, finding duplicates in streams, and related problems

by Hossein Jowhari, Mert Sağlam and Gábor Tardos

Citation. This paper addresses a question posed by Cormode et al. in VLDB 2005, namely whether a uniform (or nearly uniform) sample can be maintained in a dynamically changing database, where data items may be inserted and deleted, while using space much smaller than the size of the database. More generally, it considers maintaining an $L_{p}$ sample, where an element must be sampled with probability proportional to $w^{p}$ (possibly up to some small relative error), where $w$ is a weight that may change dynamically. In SODA 2010, Monemizadeh and Woodruff showed that it is possible to perform $L_{p}$ sampling in a stream using polylogarithmic space. The PODS 2011 paper by Jowhari, Sağlam and Tardos essentially closes the problem by presenting algorithms with improved space usage, as well as a matching lower bound showing that it is not possible to asymptotically improve the upper bounds. The paper has had a considerable impact on the design of algorithms in streaming and distributed models of computation, where $L_{p}$ sampling has become an essential part of the toolbox. The survey " $L_{p}$ Samplers and Their Applications" in ACM Computing Surveys (2019) presents a number of surprising applications, for example in graph algorithms and in randomized numerical linear algebra.

The Alberto O. Mendelzon Test-of-Time Award Committee for 2021:

\begin{abstract}
Angela Bonifati
Lyon 1 University
\end{abstract}

\author{
Rasmus Pagh \\ University of Copenhagen
}

Thomas Schwentick

TU Dortmund University 